\title{
Modeling the MOSFET's Inversion Layer and Its Universal Mobility: A New Experimental Method
}

This paper was downloaded from TechRxiv (https://www.techrxiv.org).

\section{LICENSE}

CC BY 4.0

SUBMISSION DATE / POSTED DATE

24-12-2021 / 29-12-2021

CITATION

Shoucair, F. (2021): Modeling the MOSFET's Inversion Layer and Its Universal Mobility: A New Experimental Method. TechRxiv. Preprint. https://doi.org/10.36227/techrxiv.17571509.v1

DOI

10.36227/techrxiv.17571509.v1 


\title{
Modeling the MOSFET's Inversion Layer and its Universal Mobility: A New Experimental Method ${ }^{1}$
}

\author{
F. S. Shoucair
}

Abstract-We formulate a simple, yet accurate, model for a non-uniform mobile charge density $\rho(z)$ giving rise to a mean potential $\psi^{*}$ across an inversion layer of finite extent, which we measure by means of a novel, sensitive, experimental method involving nulls of harmonic distortion components $\left(D_{2} \approx D 3 \approx 0\right)$ of the drain current under sinusoidal excitation below saturation. We thus establish analytically and experimentally, that the low-field, "universal" effective mobility $\mu_{\text {eff }}$ varies as $\sim\left(E^{*} T\right)^{-1 / 3}$ for transversal fields

$$
E_{T}^{*}=-\frac{1}{\varepsilon_{s i}}\left[\eta Q_{i}+Q_{b}\right] \leq 0.5 \mathrm{MV} / \mathrm{cm}
$$

wherein $\eta$ varies continuously between $1 / 2$ and 1/3. We also establish and observe that the higher order, derivative, parameter $\theta_{T}$ quantifying $\mu_{\text {eff }}$ 's modulation by $E^{*_{T}}$ varies as $\sim\left(E^{*} T\right)^{-5 / 3}$ under laminar flow conditions, thereby further corroborating the foregoing effects and interpretations thereof.

\section{INTRODUCTION}

The well-known charge sheet and constant effective mobility, $\mu_{\text {eff }}$, approximations, which simplify the MOSFET's (Metal Oxide Semiconductor Field Effect Transistor) drain current computations, forsake consequential information about the charge distribution $\rho(z)$ within, and mean potential $\psi^{*}$ across, the inversion layer, both of which are critical to the conceptual modeling of channel conductivity, and of the modulation thereof by transverse electric fields $E^{*} T$. The limitations and drawbacks entailed by these approximations are set forth in section II, along with their relevance to our results, which indicate that (i) the inversion layer's detailed characteristics remain consequential even as this layer shrinks with process downscaling and, thus, (ii) the conventional assumption of a "negligibly" thin inversion layer may not warrant its outright neglect, absent contrary and/or contextual justification.

In section III, we apply an experimental method originally conceived to suppress harmonic distortion components in analog integrated filters $[1,2]$, to measure the mean potential $\psi^{*}$ across the inversion layer, a novel technique in this context, which yields the corresponding mean transverse $E^{*} T$, (including $\eta$ ), as well as the ratio of mean to surface potentials $\left(\psi * / \psi_{s}\right)$ from which we deduce, and subsequently model $\rho(\mathrm{z})$ analytically in section IV. The accuracy of this model is confirmed, in section $\mathrm{V}$, by further, sensitive, measurements of the modulation of $\mu_{\text {eff }}$ by $E^{*} T$, namely the parameter $\theta_{T}=$ $(1 / \mu)\left(d \mu / d E_{T}^{*}\right)$, which are in agreement with our model's analytical predictions that $\theta_{T}$ should vary as $\sim\left(E^{*} T\right)^{-5 / 3}$. Our overall results are summarized in section VI.

\section{CONDUCTIVITY, MOBILITY, AND THE CHARGE SHEET MODEL}

Under laminar flow conditions, the conductivity of a thin sheet of charge at depth $z$ inside the inversion layer in a $(W-x)$ plane, parallel to that of the gate insulator, is $\sigma(x, z)=$ $[q \rho(x, z) \mu(z)]$, where $q$ is the electronic charge, $\rho(x, z)$ the charge density at $x$ along the channel, and $\mu(z)$ the average mobility of charge carriers

\footnotetext{
${ }^{1}$ This is a self-contained, abbreviated, version of sections III through VI (only) of the manuscript entitled "Conservation Laws at Physical Origins of Universal Mobility in MOSFET Inversion Layers in Consilience with River Flow in a Gravitational Field" (https://doi.org/10.36227/techrxiv.17088419.v1), recast in standalone form. Sections I and II are similarly archived under the title "Physical Origins of Universal Mobility in MOSFET Inversion Layers: Conservation Laws".
} 
along the $x$ direction, which is related to the drift velocity of (9) in [3]:

$$
v_{\text {drift }}=\mu(z) \frac{d V}{d x}=\mu(z) E_{I I}
$$

where $E_{/ /}$is the longitudinal electric field, along the $x$ (or channel length) direction. The resulting drain current is:

$$
\begin{gathered}
I_{D}=\left(\frac{W}{L}\right) \int_{0}^{L} \int_{0}^{z_{\max }} \sigma(x, z) d z d x \\
=\left(\frac{W}{L}\right) \int_{0}^{L} \int_{0}^{z_{\max }} q[\rho(x, z) \mu(z)] d z d x
\end{gathered}
$$

wherein the double integral is customarily simplified by assuming (a) constant mobility along the $z$ axis, $\mu(z)=\mu_{e f f}$, thereby neglecting the effects of transverse fields $E^{*} T$ on mobility and, $(b)$ an inversion layer of zero thickness the "charge-sheet" approximation — such that $\rho(x, z)=\rho(x)$ is a function of $x$ only, or of the potential $V(x)$, since $(d V / d x)=E_{/ /}$. Under these simplifying assumptions, the drain current may be expressed in closed form, by integration along the channel length:

$$
\begin{gathered}
I_{D}=\left(\frac{W}{L}\right) \mu_{e f f} \int_{0}^{V_{D}} q \rho(V) d V= \\
\mu_{e f f} C_{o x}^{\prime}\left(\frac{W}{L}\right)\left[a_{1} V_{D}+a_{2} V_{D}^{2}+a_{3} V_{D}^{3}\right] \\
+\ldots \text { higher order terms }
\end{gathered}
$$

wherein $\rho(V)$ is a known, explicit, function of the terminal voltages applied to the transistor, $V_{S}=0$ and $V_{D}$ are the potentials at its source $(x=0)$ and drain $(x=L)$ terminals respectively, $C_{o x}^{\prime}$ the gate oxide capacitance per unit area, $a 1, a 2, \ldots a n$ are constants determined by terminal bias and signal potentials, and the higher order terms are negligibly small in this context. For a given value of $I_{D}, \mu_{\text {eff }}$ is then the only unknown quantity in (3), which is thereby deduced readily from standard measurements. We note that, as simplification (a) above sets $\mu_{\text {eff }}\left(E^{*} T\right)$ $=\mu_{\text {eff }}$ in (3), the value of $E^{*_{T}}$ corresponding to a given $I_{D}$, which is essential to the determination of the function $\mu_{\text {eff }}\left(E^{*} T\right)$, is variously assumed to be an "average" [4], or an "effective" [5],[7], value $E^{*} T=-\left(1 / \varepsilon_{S i}\right)\left[\eta Q_{i}+Q_{b}\right]$, with $\eta=1 / 2$ for electrons [4],[5],[7], $\eta=1 / 3$ for holes [5], with $Q_{i}$ (corrected [8]), and $Q_{b}$, the total inversion and bulk charges, respectively.

A. Limitations of the charge-sheet and constant mobility models

In view of Poisson's potential field equation associated with a given charge distribution, the assumption of an inversion layer of zero thickness - entailing the tacit neglect of its spatial density profile - results in the loss of information about both the electric field $E(z)=(-q / \varepsilon s i) \int \rho(x, z) d z$, and the potential $\psi(z)=-\int E(z) d z$ within the inversion layer, with $\varepsilon_{S i}$ the permittivity of silicon, since these integrals are taken between identical limits $\left[0, z_{\max }(x)\right]$ when $z_{\max }=0$, as indeed the potential difference across any spatial region of zero extent is zero. The surface potential $\psi_{s}$ at the Si/insulator interface, may nonetheless be calculated accurately from knowledge of the total inversion layer charge $Q_{i}$, per (5) below, which is represented by $q \rho(V)$ in (3) above [9],[10].

If $\mu(z)$ is not assumed constant in (2), and an inversion layer of finite thickness $z_{\max } \neq 0$ is assumed, then $E(z)$ and $\psi(z)$ are, in principle, known functions given $\rho(x, z)$, but the drain current in (2) may no longer be expressed in analytical closed form as in (3), because $\mu(z)$ may not properly be removed from the integrand of (2). Consequently, and crucially for purposes of quantifying the dependence of mobility on transverse fields $\left(E^{*} T\right)$ from observations of $I_{D}$, the function $\mu_{\text {eff }}\left(E^{*} T\right)$ may no longer be "disentangled" from the result of the integration in (2), nor, thereby, extracted unambiguously from standard measurements of $I_{D}$, unless the function $\rho(x, z)$ is known to a sufficient degree of accuracy. The following section is devoted to the description of a method for achieving the latter objective. 


\section{AN ACCURATE METHOD FOR MEASURING THE MEAN \\ POTENTIAL $\left(\psi^{*}\right)$ AND TRANSVERSE ELECTRIC FIELD $\left(E^{*} T\right)$ ACROSS THE INVERSION LAYER}

The function $\rho(z)$ in (2) may be deduced from such bias conditions as result in low/null harmonic distortion components $(D 2 \approx D 3 \approx 0)$ for $I_{D}$, when sinusoidal signals of suitable amplitudes are applied to the transistor's terminals simultaneously, drain and body especially, thereby modulating $E^{*} T$, as set forth in [1],[2]: under these experimental conditions, nulls of D3 occur uniquely because substrate effects on mobility counteract those of the gate (by increasing the threshold voltage), $I_{D}$ is then (nearly) proportional to $V_{D}$, hence the channel conductance constant, and the conducting channel obeys Ohm's Law, which corresponds to laminar flow since constant $(d z / d x)=(d z / d V)(d V / d x)=(d z / d V) \mathrm{E} / /$, per II.B.(d) in [3], yields proportionality between $z(x)$ and $V(x)$, hence between $I_{D}$ and $V_{D}$.

By contrast, traditional methods [4], [5],[7], entail an extrapolation of the drain current characteristics at low drain-source potential, to a "threshold" voltage whose definition is neither well defined nor unique [9], thereby resulting in various inconsistencies. These, and other, derivative methods [8],[11],[12], have thus left unresolved the physical origins of the "universality" of mobility curves, among various related observations.

The method implemented below sidesteps such inaccuracies and inconsistencies as incurred by the latter methods, by setting forth the objective criterion $(D 2 \approx D 3 \approx 0)$ for determining the mean electric field $E^{*} T$ across the inversion layer from measurements of $\psi^{*}$. This method furthermore yields $\rho(z), \mu_{\text {eff }}\left(E^{*} T\right)$, and the latter' derivative function $\left(\theta_{T}\right)$ accurately, thereby rendering these quantities in a manner that is mutually consistent, and verifiable by direct observation, as will be shown in the sequel.

\section{A. Measuring the mean potential $\psi^{*}$ across the inversion layer}

In the ensuing, we use standard MOSFET terminology for an $\mathrm{n}$ channel device, and denote $V_{B}=\left(V_{S B}+2 \phi_{F}+b^{*} \phi_{t}\right)$, wherein $\phi_{F}$ and $\phi_{t}=k T / q$ are the Fermi and thermal potentials respectively. The mean potential $\psi^{*}$ across the inversion layer is:

$$
\Psi^{*}=b^{*} \Phi_{t}=\ln \left[\left(\frac{2}{\gamma}\right) \sqrt{V_{B}}\left(\frac{1}{r}-1\right)\right]
$$

where $r=\Delta V_{B} / \Delta V_{G S}$ is the ratio of increments of applied $V_{B}$ and $V_{G S}$ bias voltages which result in observed nulls of $D 3$, per (3) in [1], and $\gamma$ is the body effect coefficient [9]. The mean, observed, value of $r$ over the range of our data is 0.39 . The corresponding, observed, values of $\psi^{*}=\left(b^{*} \phi t\right)$ range between $\left(1.45 \phi_{t}\right)$ and $\left(1.65 \phi_{t}\right)$, (see Fig.1), and the analytical values of the surface potential $\psi_{s}=\left(b_{s} \phi_{t}\right)$ range between $\left(4.5 \phi_{t}\right)$ and $\left(6.7 \phi_{t}\right)$, as obtained by solving the transcendental [9]:

$$
\begin{gathered}
\Psi_{S}=V_{G S}-V_{T 0}+\gamma \sqrt{2 \Phi_{F}}- \\
\gamma \sqrt{\Psi_{s}+\Phi_{t}\left[e^{\left(\Psi_{s}-V B+V\right) / \Phi_{t}}-1\right]}
\end{gathered}
$$

for $\left(\psi_{s}-V_{B}\right)=\left(b_{s} \phi_{t}\right)$ to $0.1 \%$ accuracy for the observed bias conditions, with $V=0$. The ratio of derivatives,

$$
\left(\frac{\partial \psi_{s}}{\partial V_{G S}}\right) /\left(\frac{\partial \psi_{s}}{\partial V_{B}}\right)=r
$$

yields the right-hand side of (4), which relates $r$ to $b^{*}$, thereby determining $b^{*}$ and $\psi^{*}$. The observed variations of $\psi^{*}$, and our analytical predictions for these (section IV below), are shown in Fig.1 for ready comparison with the empirical Schwarz-Russek model [7].

The observed ratio $\left(\psi^{*} / \psi_{s}\right)$ thus ranges between $(1.45) /(4.5) \approx(1 / 3)$ near to the onset of strong inversion, and $(1.65) /(6.7) \approx(1 / 4)$ near to the saturation of $\psi_{s}$ (see Fig. 2). A ratio of (1/3) is expected from Poisson's equation for a spatially uniform charge distribution, whereas a ratio of (1/4) corresponds to a spatially linear distribution $\rho(z) \sim z$. These 
results are suggestive of the analytical model to follow, which must account for the foregoing observations.

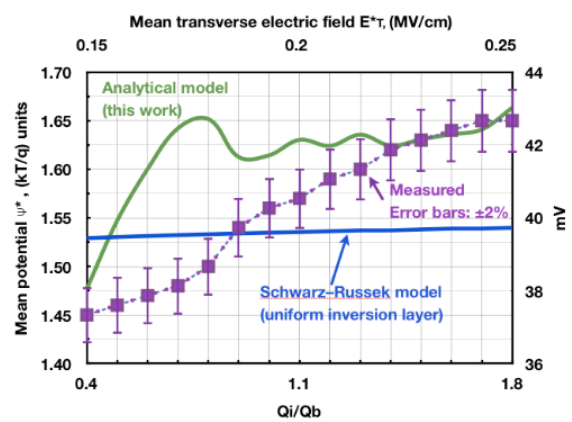

Fig. 1 Observed mean potential $\psi^{*}$ across inversion layer and predictions of (13).

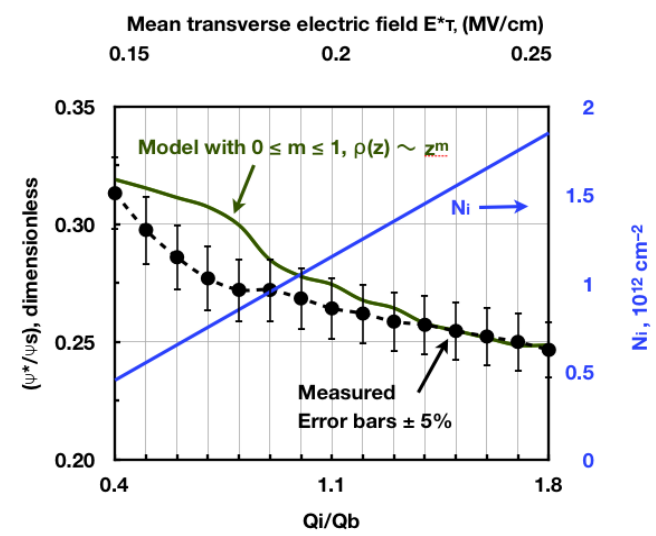

Fig. 2 Observed ratio $\left(\psi^{*} / \psi_{s}\right)$ and carrier concentration of commensurate charge sheet model $\left(N_{i}=Q_{i} / q\right)$. Model curve is per (13).

B. Determining the mean transverse electric field $E^{*}$ across the inversion layer

The mean electric field $E^{*}(V)$, which is modulated by applied, sinusoidal, drainsource and body-source potentials [1,2], is obtained from $Q_{i}\left(\psi_{s}\right)$ and $Q_{b}\left(\psi_{s}\right)$ [9, p.79]:

$$
\begin{gathered}
Q_{i}\left(\Psi_{s}\right) \approx-\gamma C_{o x}^{\prime}\left[\sqrt{\Psi_{s}+\Phi_{t} e^{\left(\Psi_{s}-V B+V\right) / \Phi_{t}}}-\sqrt{\Psi_{s}}\right](\mathbf{6}) \\
Q_{b}\left(\Psi_{s}\right) \approx-\gamma C_{o x}^{\prime} \sqrt{\Psi_{s}+V}
\end{gathered}
$$

wherein $C^{\prime}{ }_{\text {ox }}$ is the gate oxide capacitance per unit area. The mean transverse electric field is then:

$$
\begin{aligned}
E_{T}^{*} & \approx-\frac{1}{\varepsilon_{s i}}\left[\frac{Q_{i}\left(\Psi_{s}\right)}{m\left(E_{T}^{*}\right)+2}+Q_{b}\left(\Psi_{s}\right)\right]= \\
& -\frac{1}{\varepsilon_{s i}}\left[\eta\left(E_{T}^{*}\right) Q_{i}\left(\Psi_{s}\right)+Q_{b}\left(\Psi_{s}\right)\right]
\end{aligned}
$$

wherein $0 \leq m\left(E^{*}\right) \leq 1$ is the power of $z$ in $\rho(z) \sim z^{m}$ per (7) in [3], and to which corresponds $(1 / 3) \leq \eta\left(E_{T}^{*}\right) \leq(1 / 2)$, per (8) above. The correspondence between the values of $m$ and $\eta$ extracted from our measurements of $\left(\psi^{*} / \psi_{s}\right)$, and the mean values of $z$ resulting for the inversion layer are shown in Fig. 3 below, wherein:

(a) $m\left(E^{*} T\right)$ varies approximately linearly with $\left(Q_{i} / Q_{b}\right)$ near to $\left(Q_{i} / Q_{b}\right) \approx 1$; i.e., for $E_{T}^{*} \approx 0.2 \mathrm{MV} / \mathrm{cm}$.

(b) $\eta\left(E^{*}\right)=1 /\left[m\left(E^{*} T\right)+2\right]$ is a related function of inversion level $\left(Q_{i} / Q_{b}\right)$.

(c) the ratio $\left(\psi^{*} / \psi_{s}\right)$ varies between $(1 / 3)$ and (1/4) (as per Fig.2 above).

(d) the mean inversion layer thickness $\langle z\rangle=\left(\psi^{*} / E^{*} T\right)$ resulting from our observations of $\psi^{*}$ and $E^{*}{ }_{T}$ (per Fig.1) is commensurate with the predictions of Stern and Howard [13], and Schwarz and Russek [7].

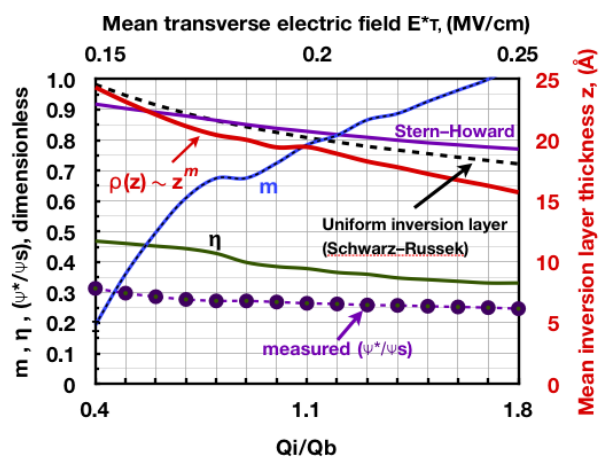

Fig. 3 Observed values of $m,\left(\psi^{*} / \psi_{s}\right)$, and $\langle z>$.

$$
\begin{aligned}
& \text { IV. ANALYTICAL MODEL OF THE } \\
& \text { INVERSION LAYER'S CHARGE } \\
& \text { DENSITY PROFILE DEDUCED FROM } \\
& \text { EXPERIMENTAL OBSERVATIONS }
\end{aligned}
$$

In view of the observations described in section III. $A$ above we posit, for an 
inversion layer of extent $z_{\max }$ supporting a given total charge $Q_{c}=Q_{i}+Q_{b}$ :

$$
b(z) \approx b^{*}\left(\frac{z}{z_{\max }}\right)^{m} ; 0 \leq z \leq z_{\max }
$$

such that $\psi^{*}=b^{*} \phi_{t}$ represents the mean potential across $z_{\max }$, since $b\left(z_{\max }\right)=b^{*}$ when $z=z_{\max }$ and, ignoring minority holes,

$$
\rho(z) \approx N_{a}\left[e^{b^{*}\left(\frac{z}{z_{\max }}\right)^{m}}-1\right]
$$

for the corresponding mobile charge density within the inversion layer, pending experimental corroboration. Equation (10) is equivalent to a linear approximation $\rho(z) \sim z$ when $m \approx 1$ and $\left(z / z_{\max }\right)<<1$ (see Figs.4(b) and (c) below), which meets the critical boundary condition $\rho(0)=0$.

(a)

(b)
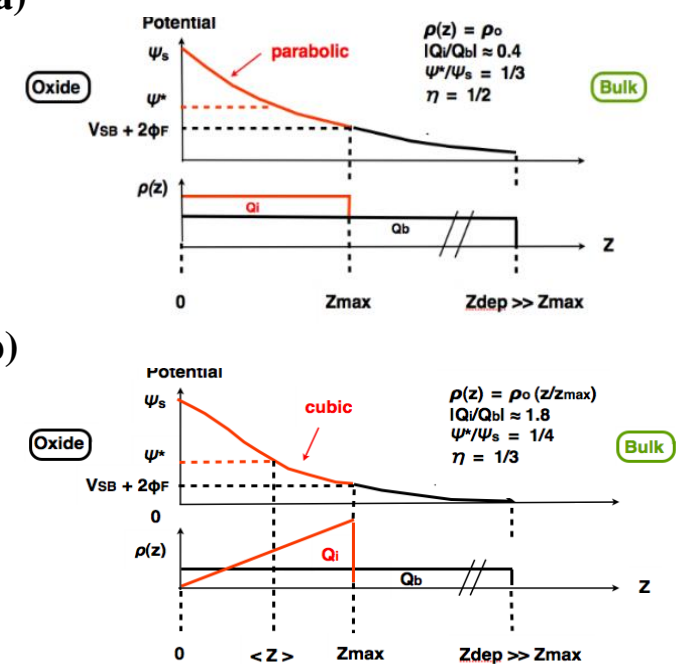

(c)
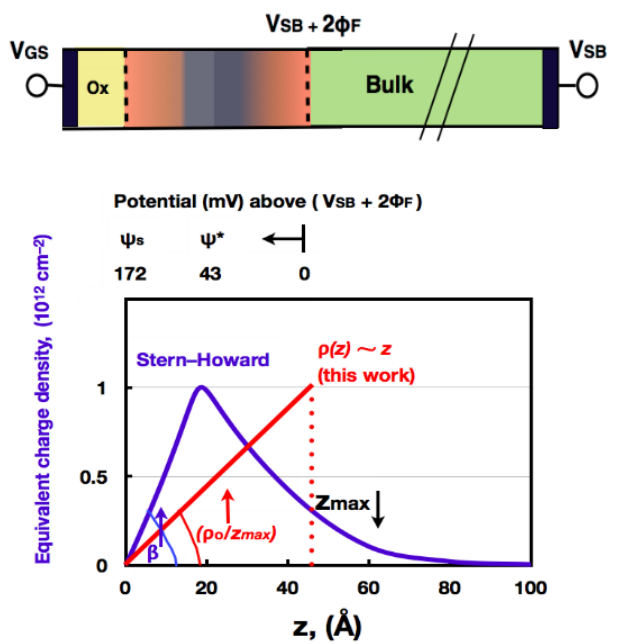

Fig. 4(a) Uniform inversion layer charge density; $\left|Q_{i} / Q_{b}\right| \approx 0.4, \eta=(1 / 2),\left(\psi^{*} / \psi_{s}\right)=(1 / 3), \psi^{*}=b^{*} \phi_{t} \approx$ $1.45 \phi_{t} \approx 37 \mathrm{mV}, \psi_{s} \approx 111 \mathrm{mV}$ (see Figs.1 and 2) observed at low values of $\left|Q_{i} / Q_{b}\right|<1$ where the "flat" depletion charge $Q_{b}$ is dominant. The standard "flat" inversion charge profile between 0 and $z_{\max }$ becomes physically unrealistic as $\left|Q_{i} / Q_{b}\right|$ increases, because there must be a finite transition region near to the oxide interface wherein $\rho(z)$ drops towards zero, which is a critical boundary condition of the system.

(b) $\left|Q_{i} / Q_{b}\right| \approx 1.8, \eta=(1 / 3),\left(\psi^{*} / \psi_{s}\right)=(1 / 4), \psi^{*}=b^{*} \phi_{t} \approx$ $1.65 \phi_{t} \approx 43 \mathrm{mV}, \psi_{s} \approx 172 \mathrm{mV}$; unlike in (a), the boundary condition $\rho(z=0)=0$ holds for our model which is here a valid approximation of:

(c) Stern-Howard model [13] for an inversion layer charge density $\rho(z) \sim(1 / 2) \beta^{3} z^{2} e^{-\beta z}, \beta \approx(1 / 15)$, whose $\langle z\rangle \approx(3 / 2 \beta) \approx 22.5 \AA$, and $z_{\max } \approx 45 \AA$. The areas, between 0 and $z_{\max }$, under the Stern-Howard curve and our model's are nominally equal, and correspond to the total inversion charge $Q_{i}$. The potential values indicated correspond to condition (b).

Equating the inversion charge of the sheet model, $Q_{i}\left(\psi_{s}\right)$ per (6), to that posited by (10) for an inversion layer of finite extent $z_{\max }$,

$$
\begin{aligned}
Q_{i}=\int_{0}^{z_{\max }} q \rho(z) d z \approx & \\
& \int_{0}^{z_{\max }} q N_{a}\left[e^{b^{*}\left(\frac{z}{z_{\max }}\right)^{m}}-1\right] d z
\end{aligned}
$$

establishes an implicit analytical relationship between the surface potential $\psi_{s}=b_{s} \phi t$ of the sheet model, per (5), and the desired mean potential $\psi^{*}=b^{*} \phi_{t}$ derived from (11). Integrating Poisson's equation with $\rho(z)$ thus chosen, twice to obtain the surface potential $\psi_{s}=\left(b_{s} \phi_{t}\right)$, once more to obtain the mean potential $\psi^{*}=\left(b^{*} \phi_{t}\right)$ over $\left[0, z_{\max }\right]$, thus yields:

$$
\frac{\Psi^{*}}{\Psi_{s}}=\frac{b^{*}}{b_{s}}=\frac{1}{m+3}
$$

whereby $\eta=1 /(m+2)$ for $E^{*} T$ in (8), which is obtained as an intermediate result.

For $0 \leq m\left(E^{*} T\right) \leq 1$, the parameter $\eta$ is thus expected to vary continuously between (1/2) and (1/3), per (8), as observed for standard devices with $\langle 100\rangle$ surfaces, and predictably as a function of inversion level (see Fig.3). Because this transition is especially prominent in the range of bias conditions whereby neither $Q_{i}$ nor $Q_{b}$ is overwhelmingly dominant over the other, our 
measurements are concentrated on this particular range, namely $0.4 \leq\left(Q_{i} / Q_{b}\right) \leq 1.8$ (see Figs. 1-3,5), which corresponds, approximately, to $0.15 \mathrm{MV} / \mathrm{cm} \leq E^{*}{ }_{T} \leq 0.25$ $\mathrm{MV} / \mathrm{cm}$, where laminar flow obtains, and over which we observe $D 3$ nulls.

form:

Proceeding thus, we derive in closed

$$
\frac{b^{*}}{b_{s}}=\frac{e^{b^{*}}-1-b^{*}-\frac{\left(b^{*}\right)^{2}}{2 !}-\frac{\left(b^{*}\right)^{3}}{3 !}}{b^{*}\left(e^{b^{*}}-1-b^{*}-\frac{\left(b^{*}\right)^{2}}{2 !}\right)}
$$

which yields $b^{*}$ readily, given $b_{s}=\left(\psi_{s} / \phi_{t}\right)$ as obtained from (5). The ratio (13) thus reduces to the expected value $(3 !) /(4 !)=(1 / 4)$ in the charge-sheet limit $b^{*} \rightarrow 0$, and matches observations of $\left(b * / b_{s}\right)=\left(\psi^{*} / \psi_{s}\right)$ nearly perfectly for $\left(Q_{i} / Q_{b}\right) \geq 1$, where $m \approx 1$ as observed in Fig.2, and otherwise to within $\pm 5 \%$, on an average, over the full observation span. The left-hand side of (13) indicates that the ratio $\left(b * / b_{s}\right)=\left(\psi^{*} / \psi_{s}\right)$ decreases as $\psi_{s}$ increases, i.e., as inversion level increases: physically, this means that as $E^{*} T$ increases, the increasing electron charge contributes mainly to an inversion layer's mobile $Q_{i}$ concentrated nearer to the oxide, while the fixed depletion charge $Q_{b}$ remains relatively unaltered. These trends are indicated by the arrows attached to the various quantities depicted in Fig.4(c).

The ratio of mean electric field $\left(E^{*}\right)$ across the inversion layer corresponding to $\rho(z)$ per $(10)$, to that $\left(E^{*}{ }_{u}\right)$ of a uniform charge distribution supporting the same total charge $Q_{c}$, and obtained as an intermediate result is:

$$
\frac{E_{\rho}^{*}}{E_{u}^{*}}=\left(\frac{2}{b^{*}}\right)\left[\frac{e^{b^{*}}-1-b^{*}-\frac{\left(b^{*}\right)^{2}}{2 !}}{e^{b^{*}}-1-b^{*}}\right]
$$

whose charge-sheet limit is indeed $(2 / 3)$, as expected when $b^{*}$ approaches 0 :

$$
\lim _{b^{*} \rightarrow 0}\left(\frac{E_{\rho}^{*}}{E_{u}^{*}}\right)=\left(\frac{2}{b^{*}}\right)\left[\frac{\frac{\left(b^{*}\right)^{3}}{3 !}}{\frac{\left(b^{*}\right)^{2}}{2 !}}\right]=\frac{2}{3}
$$

\footnotetext{
2 Although $\theta_{T}$ may likewise be obtained from nulls of
} $D_{2}$, those of $D_{3}$ are much more sensitive to bias and in which case the mean transverse electric field $E^{*} T$, per (8), is:

$$
-\varepsilon_{s i} E_{T}^{*}=\frac{Q_{i}}{2}\left(\frac{E_{\rho}^{*}}{E_{u}^{*}}\right)+Q_{b}
$$

wherein the ratio $\left(E^{*}{ }_{\rho} / 2 E^{*}\right)=\eta$ yields the expected result $(1 / 2) \cdot(2 / 3)=(1 / 3)$ for an inversion layer varying linearly with $z$, or $m=1$ in (9)-(12), as indicated above. The traditional assumption of a value $\eta=(1 / 2)$, fixed a priori (as illustrated in Fig.4(a), and corresponding to constant $\rho(z)$ ), which does not account for these effects, and fails to meet the boundary condition $\rho(0)=0$, may thereby be expected to give rise to observable discrepancies left wanting of clarification and reconciliation, as indeed noted in [4],[5],[7].

\section{TRANSVERSE-FIELD MODULATION PARAMETER $\left(\theta_{T}\right)$}

Conceptually, $\theta_{T}$ represents a first order Taylor series expansion of the expression for the mobility $\mu_{\text {eff }}\left(E^{*} T\right)$ associated with the MOSFET's drain current: the constant $\mu_{\text {eff }}$ in (3) is replaced by $\mu_{\text {eff }}\left[1+\theta_{T} \cdot V(x)\right]$, where $V(x)$ is the potential along the channel's direction $(x)$, and $\left[\theta_{T} \cdot V(x)\right]<<1$. The drain current $I_{D}$ is then obtained by integrating the function $\left[1+\theta_{T} \cdot V(x)\right] \cdot \rho[V(x)]$ from $O$ to $L$, in the conventional manner [1],[2].

\section{A. Experimental conditions and results}

All measurements were made on long n-channel devices $(L \approx 100 \mu \mathrm{m})$ fabricated in a standard, industrial, CMOS process. The range of $E^{*} T$ for our data is centered near to $0.2 \mathrm{MV} / \mathrm{cm}$, where universal mobility behavior prevails, as reported in the literature [4],[5].

$\theta_{T}$ was extracted from nulls of third harmonic current distortion components $(D 2 \approx 0, D 3=0)^{2}$ in the triode region of operation, with applied $V_{D S}=V_{\text {peak }} \sin (2 \pi f t)$, where $V_{\text {peak }}<V_{D S s a t}, f=1 \mathrm{kHz}$, and $\left(E^{*} T / E_{/ /}\right)$ $\geq 2000$, [1]:

signal amplitude conditions (see II. $B$ in [1]), which is an advantage for the purpose at hand. 


$$
\theta_{T}\left(E_{T}^{*}\right)=\left(\frac{\gamma}{8}\right)\left[\frac{V_{B}^{-\frac{3}{2}}}{1-\frac{\gamma}{4 \sqrt{V_{B}}}}\right]
$$

Fig.5 below compares these observations to the predictions of the foregoing model, as set forth in section III. $B$, whereby $E^{*} T$ is as per (8):

$$
\begin{gathered}
\left.\theta_{T}(V)\right|_{V=0}=\left.\left(\frac{1}{\mu}\right) \frac{d \mu}{d V}\right|_{V=0}=\left.\left(\frac{1}{\mu}\right) \frac{\partial \mu}{\partial E_{T}^{*}} \frac{d E_{T}^{*}}{d V}\right|_{V=0} \\
\approx \frac{1}{3}\left[\frac{1-\frac{\gamma}{2 \sqrt{V_{B}}}\left(\frac{1}{\eta}-1\right)}{V_{G S}-V_{T 0}+\gamma\left(\sqrt{V_{B}}+\sqrt{\left.2 \Phi_{F}\right)}\right.}\right]
\end{gathered}
$$

While the agreement of (18) is within $\approx \pm 5 \%$ throughout the observation range, the qualitative effect of varying values of $m$ (hence of $\eta$ ) is most conspicuous, which illustrates the inadequacy of a uniform inversion layer model (the curve labeled $m=0$, or fixed $\eta=1 / 2$ ): the data deviate from the latter, increasingly as $m$ increases between 0.2 and 1.05 (i.e., $\eta$ decreasing between (1/2) and (1/3) approximately, as also illustrated in Fig.5), whereas (18) accounts for these effects while rendering $\theta_{T}$ explicitly in terms of terminal potentials and device parameters.

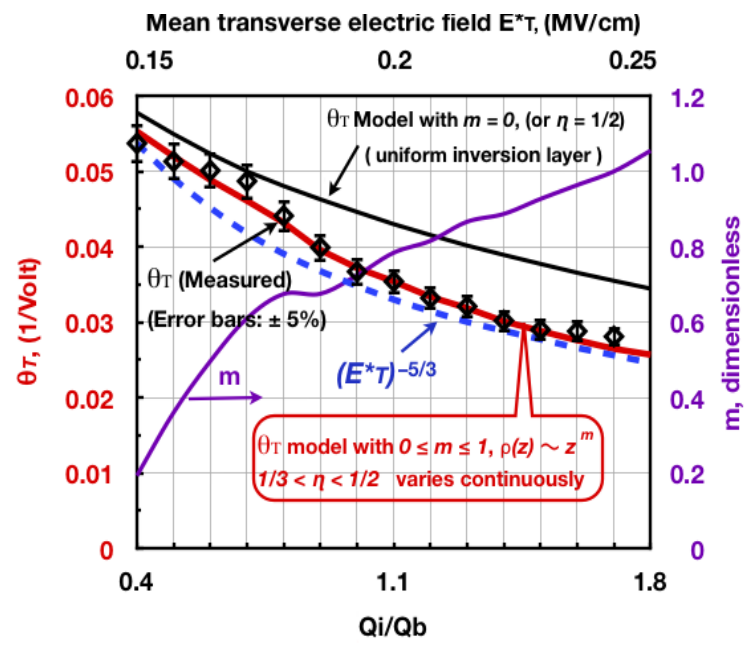

Fig. 5. Parameter $\theta_{T}$, defined by (18), quantifying mobility modulation by $E^{*} T$. Our data are in good agreement with the expected behavior per (19), whereas the model with fixed $\eta=1 / 2$ is not.

A further, crucial, check on the consistency of the foregoing results, and confirmation of laminar flow conditions, are now at hand: since, under such conditions, $\mu_{\text {eff }}$ and $\langle z\rangle$ vary as $\sim\left(E^{*} T\right)^{-1 / 3},(d z / d x) \approx-\left(E_{/ / /} E^{*} T\right)$ per (11) in [3], $\psi^{*}$ is a weak function of $z$ (Fig.1), and $(d x / d V)=\left(1 / E_{/ /}\right)$, we expect:

$$
\begin{gathered}
\theta_{T}(V)=\left(\frac{1}{\mu}\right) \frac{d \mu}{d V}=\left(\frac{1}{\mu} \frac{\partial \mu}{\partial E_{T}^{*}}\right) \frac{\partial E_{T}^{*}}{\partial \Psi^{*}}\left(\frac{\partial \Psi^{*}}{\partial z}\right) \frac{\partial z}{\partial x} \frac{d x}{d V} \\
\sim\left(\frac{-1}{3 E_{T}^{*}}\right) \frac{1}{<z>}\left(\frac{-E_{I I}}{E_{T}^{*}}\right) \frac{1}{E_{I I}} \sim \frac{\left(E_{T}^{*}\right)^{\frac{1}{3}}}{\left(E_{T}^{*}\right)^{2}} \sim\left(E_{T}^{*}\right)^{-\frac{5}{3}}
\end{gathered}
$$

which is indeed observed in Fig. 5. In view of our analogy with a river's flow [3], the same result may be deduced from (4) and/or (9) in [3], wherein $v d v=-z d z$, with $v=\left(\mu E_{/ /}\right)$from (1) above, such that:

$$
\begin{gathered}
\frac{1}{\mu} \frac{d \mu}{d E_{T}^{*}}=\frac{1}{v} \frac{d v}{d E_{T}^{*}}=-z \frac{d z}{d E_{T}^{*}} \\
\sim-\left(E_{T}^{*}\right)^{-\frac{1}{3}} \cdot \frac{d}{d E_{T}^{*}}\left[\left(E_{T}^{*}\right)^{-\frac{1}{3}}\right] \sim-\left(E_{T}^{*}\right)^{-\frac{1}{3}} \cdot\left[-\left(E_{T}^{*}\right)^{-\frac{4}{3}}\right]
\end{gathered}
$$

\section{SUMMARY}

A sensitive method for measuring the mean potential $\left(\psi^{*}\right)$ across the MOSFET's inversion layer was applied under conditions of harmonic distortion component nulls $(D 2 \approx D 3 \approx 0$ ) of the drain current under sinusoidal excitation, below saturation. We showed, thereby, how the inversion charge density profile $\rho(z)$ may be deduced accurately from the ratio $\left(\psi * / \psi_{s}\right)$, as the surface potential $\left(\psi_{s}\right)$ increases from onset of strong inversion towards saturation, and how such information underpins the physical origins of disparate observations of long standing. We found and established:

(1) Non-uniform, or non-constant, $\rho(z)$ causes variations of $1 / 3 \leq \eta \leq 1 / 2$, which determine the mean transverse electric field $E^{*} T=-\left(1 / \varepsilon_{S i}\right)\left[\eta Q_{i}+Q_{b}\right]$ in terms of the inversion $Q_{i}$, and bulk $Q_{b}$, charges.

(2) The higher order, derivative, parameter $\theta_{T}$ quantifying the modulation of $\mu_{e f f}$ by $E^{*} T$ 
varies as $\sim\left(E^{*} T\right)^{-5 / 3}$ under laminar flow conditions.

\section{ACKNOWLEDGMENT}

The author is indebted to Mrs. V. Vigil, Berkeley Public Library, Berkeley, CA, and to R. Rice, for valuable technical discussions and assistance.

\section{REFERENCES}

[1] F. S. Shoucair and W. R. Patterson, "Analysis and Modeling of nonlinearities in VLSI MOSFETs including substrate effects", IEEE Trans. Electron Devices, vol. ED-40, no.10, pp. 1760-1767, October 1993.

[2] F. S. Shoucair , "Modeling, decoupling and suppression of MOSFET distortion components", IEE Proceedings, Circuits, Devices and Systems, vol. ED-146, no.1, pp. 37-43, February 1999.

[3] F. S. Shoucair , "Physical Origins of Universal Mobility in MOSFET Inversion Layers: Conservation Laws", unpublished. [Online]. Available:

https://doi.org/10.36227/techrxiv.17088419.v1.

[4] S. C. Sun and J.D. Plummer, "Electron mobility in inversion and accumulation layers on thermally oxidized silicon surfaces", IEEE Trans. Electron Devices, vol. ED-27, no.8, pp. 1497-1508, August 1980.

[5] S. Takagi, A. Toriumi, M. Iwase, and H. Tango, "On the universality of inversion layer mobility in $\mathrm{Si}$ MOSFET's: Part - I Effects of substrate impurity concentration, Part - II, Effects of surface orientation", IEEE Trans. Electron Devices, vol. ED-41, no.12, pp. 2357-2368, December 1994.

[6] S-W. Lee, "Extraction of MOSFET carrier mobility characteristics and calibration of a mobility model for numerical device simulation", Solid-State electronics, vol. 33, no. 6, pp. 719-726, 1990

[7] S. A. Schwarz and S. E. Russek, "Semi-empirical equations for electron velocity in Silicon: Part II MOS inversion layers ", IEEE Trans. Electron Devices, vol. ED-30, no.12, pp. 1634-1639, December 1983.

[8] W. Zhu, J-P. Han, and T.P. Ma, "Mobility measurement and degradation mechanisms of MOSFETs made with ultrathin high-k dielectrics", IEEE Trans. Electron Devices, vol. ED-51, no.1, pp.98-105, January 2004.

[9] Y. P. Tsividis, Operation and Modeling of the MOS Transistor, McGraw-Hill, 1987, p.79.

[10] S. M. Sze, Physics of Semiconductor Devices, John Wiley \& Sons, 1981, p.850.

[11] K. Remachan, N.A. Wong, K. Chan, S.P. Sim and C.Y. Yang, "Modeling inversion-layer carrier mobilities in all regions of MOSFET operation ", Solid-State electronics, vol. 46, pp. 153-156, 2002.

[12] P-M.D. Chow and K-L. Wang, "A new AC technique for accurate determination of channel charge and mobility in very thin gate MOSFET's", IEEE Trans. Electron Devices, vol. ED-33, no.9, pp.1299-1304, September 1986.
[13] F. Stern and W.E. Howard, "Properties of semiconductor surface inversion layers in the electric quantum limit", Physical Review, vol. 163, no.3, pp. 816-835, November 1967. 